\title{
LEARNING DISABILITIES
}

FOCAL CEREBRAL DYSFUNCTION AND LEARNING DISABILITIES

Single photon emission tamography was used to study regional

cerebral activity in 24 children with developmental learning disabilities and 15 age matched controls at the John F. Kennedy Institute, Glostrup, and Department of Neurology, Rigshospitalet, Copenhagen, Denmark. The distribution of regional cerebral activity was abnormal - low in striatal and posterior periventricular regions and high in occipital regions - in nine children with pure attention deficit and hyperactivity disorder, low in striatal and posterior ventricular areas in eight children with ADHD plus phonologicsyntactic dysphasia, and low in the left temporofrontal regions in seven children with dysphasia wi thout hyperactive behavior. (Lou HC et al. Focal cerebral dysfunction in developmental learning disabilities. Lancet Jan 6, 1990; $335: 8-11)$.

COMMENT. The use of PET in children is restricted by the radiation dose and invasive procedure. The smaller dose of radiation associated with SPET was considered less invasive and hazardous. The MRI avoids the risk of radiation side effects and has illuminated structural cerebral defects underlying various learning disabilities, e.g. cortical heterotopias in dyslexic patients, and temporal lobe cysts in children with auditory perceptual problems. The regional cerebral blow flow abnormalities demonstrated by SPET may reveal focal cerebral dysfunction not demonstrated by MRI but correlating with expressive language dysfunction and other developmental learning disabilities.

SOFT NEUROLOGICAL SIGNS IN MALNOURISHED CHILDREN

The relation of abnormal soft neurologic signs and EEG abnormalities to the severity of malnutrition was investigated in 208, 8-10 year old male school children at the Nutrition Section, Department of Paediatrics, and the Section of Neurology, Department of Medicine, Institute of Medical Sciences, Banaras Hindu University, Varanasi, India. No child had a history of birth anoxia, head injury, or drug ingestion. Seven motor tasks were tested for soft neurologic signs: 1) Finger tapping, 2) successive finger movements, 3) toe tapping, 4) heel toe tapping, 5) repetitive hand patting, 6) alternating hand pronation supination, 7) alternating hand flexion extension. Both dominant and nondominant limbs were evaluated. Choreoathetoid movements were examined as a child walked 20 steps on inverted feet (Fog test). There was a strong correlation between nutritional status and performance of motor tasks in both hands and a progressive increase in abnormalities with increased severity of malnutrition. Of 71 children with normal nutritional status $4.2 \%$ were positive for soft neurological signs whereas in 79 children with moderate malnutrition $87.3 \%$ were positive for neurological abnormalities and $50 \%$ showed a positive Fog test with choreoathetoid movements. The EEG pattern in 16 children with soft neurological signs showed abnormalities in the 